\title{
FINITENESS OF DISJOINT MINIMAL GRAPHS
}

\author{
PETER Li ${ }^{1}$ AND JiAPING WANG ${ }^{2}$
}

Let $\Omega \subset \mathbb{R}^{n}$ be an open subset of $\mathbb{R}^{n}$ with boundary given by $\partial \Omega$. Suppose $u$ is a solution to the minimal surface equation

$$
\operatorname{div}\left(\frac{\nabla u}{\sqrt{1+|\nabla u|^{2}}}\right)=0
$$

defined on $\Omega$ satisfying $u=0$ on $\partial \Omega$. The graph

$$
G=\{(x, u(x)) \mid x \in \Omega\}
$$

of $u$ in $\mathbb{R}^{n+1}$ is called a minimal graph supported on $\Omega$.

In a recent article of Meeks-Rosenberg [M-R], where they proved the unicity of the helicoid, the authors showed that if the defining functions $\left\{u_{i}\right\}$ of a set of disjointly supported minimal graphs $\left\{G_{i}\right\}$ have bounded gradients, then the number of graphs must be finite. In a private communication with the first author, Rosenberg posed the question if the number of disjoint minimal graphs, whose defining functions are at most polynomial growth of a fixed degree, is finite. Obviously, this question was motivated by his work with Meeks, but it was also related to the type of finiteness theorems the first author proved in [L] concerning harmonic functions. This argument was later generalized by the authors $[\mathrm{L}-\mathrm{W}]$ to show the finiteness of disjoint $d$-massive sets and was used to prove a structural theorem for harmonic maps. It turns out that this argument can also be used to study disjoint minimal graphs. Indeed, the purpose of this note is to show that there are only finitely many minimal graphs supported on disjoint open subsets in $\mathbb{R}^{n}$. Moreover, we will prove that the maximum possible number of such disjointly supported minimal graphs is $(n+1) 2^{n+1}$. We would like to point out that it is somewhat surprising that the finiteness theorem is valid without any assumption on the growth rate of the defining functions.

After we communicated with Rosenberg of our result, he informed us that Spruck has recently proved that the number of disjoint sublinear growth minimal graphs is at most 2. However, we have not been able to obtain a preprint

\footnotetext{
${ }^{1}$ Research partially supported by NSF grant \#DMS-9971418 and an Earmarked grant of Hong Kong.

${ }^{2}$ Research partially supported by NSF grant \#DMS-0072181.

Received August 1, 2001.
} 
from Spruck directly and hence is unable to state his theorem precisely. In any case, our estimate is less sharp when restricted to the special case of sublinear growth minimal graph, and it seems an interesting question to obtain the precise estimate for the general case. In this direction, Earp and Rosenberg [E-R] have shown that the angle of a wedge in $\mathbb{R}^{2}$ must be at least $\pi$ in order for it to support a nontrivial minimal graph.

The first author would like to express his gratitude to Harold Rosenberg for bringing this question to his attention and his encouragement for writing up this result.

In the following, we denote by $G(R)$, the extrinsic ball of radius $R$ centered at the origin of $\mathbb{R}^{n+1}$, given by

$$
G(R)=G \cap B^{n+1}(R)
$$

where $B^{n+1}(R)$ is the Euclidean $(n+1)$-ball centered at the origin of $\mathbb{R}^{n+1}$. The key lemma is to establish that a minimal graph must have Euclidean volume growth.

Lemma 1. The n-dimensional volume of $G(R)$ must satisfy the upper bound

$$
V(G(R)) \leq V\left(\Omega \cap B^{n}(R)\right)+\frac{1}{\delta} V\left(\Omega \cap\left(B^{n}((1+\delta) R) \backslash B^{n}(R)\right)\right)
$$

for any $\delta>0$, where $B^{n}(r)$ denotes the radius $r$ ball centered at the origin in $\mathbb{R}^{n}$. In particular,

$$
V(G(R)) \leq(n+1) \omega_{n} R^{n} .
$$

Proof. For $R>0$, let us define the function

$$
u_{R}(x)=\left\{\begin{array}{rll}
R & \text { if } & u>R \\
u(x) & \text { if } & |u(x)| \leq R \\
-R & \text { if } & u<-R .
\end{array}\right.
$$

For any $\delta>0$, let $\phi$ be a non-negative cut-off function defined on $\mathbb{R}^{n}$ given by

$$
\phi=\left\{\begin{array}{ccl}
1 & \text { on } & B^{n}(R) \\
\frac{(1+\delta) R-r(x)}{\delta R} & \text { on } & B^{n}((1+\delta) R) \backslash B^{n}(R) \\
0 & \text { on } & \mathbb{R}^{n} \backslash B^{n}((1+\delta) R),
\end{array}\right.
$$

where $r(x)$ is the Euclidean distance to the origin of $\mathbb{R}^{n}$. Note that $\phi u_{R}=0$ on $\partial\left(\Omega \cap B^{n}((1+\delta) R)\right)$. Integration by parts yields

$$
\begin{aligned}
0 & =\int_{\Omega \cap B^{n}((1+\delta) R)} \phi u_{R} \operatorname{div}\left(\frac{\nabla u}{\sqrt{1+|\nabla u|^{2}}}\right) \\
& =-\int_{\Omega \cap B^{n}((1+\delta) R)} \frac{\phi\left\langle\nabla u_{R}, \nabla u\right\rangle}{\sqrt{1+|\nabla u|^{2}}}-\int_{\Omega \cap B^{n}((1+\delta) R)} \frac{u_{R}\langle\nabla \phi, \nabla u\rangle}{\sqrt{1+|\nabla u|^{2}}} .
\end{aligned}
$$


Using the facts that $|\nabla \phi| \leq \frac{1}{\delta R}$ and $\left|u_{R}\right| \leq R$, we have

$$
\begin{aligned}
\int_{\Omega \cap B^{n}((1+\delta) R)} \frac{\phi\left\langle\nabla u_{R}, \nabla u\right\rangle}{\sqrt{1+|\nabla u|^{2}}} & \leq \int_{\Omega \cap B^{n}((1+\delta) R)} \frac{|\nabla \phi|\left|u_{R}\right||\nabla u|}{\sqrt{1+|\nabla u|^{2}}} \\
& \leq \frac{1}{\delta} V\left(\Omega \cap\left(B^{n}((1+\delta) R) \backslash B^{n}(R)\right)\right) .
\end{aligned}
$$

In particular,

$$
\int_{\Omega \cap B^{n}(R) \cap\{|u| \leq R\}} \frac{|\nabla u|^{2}}{\sqrt{1+|\nabla u|^{2}}} \leq \frac{1}{\delta} V\left(\Omega \cap\left(B^{n}((1+\delta) R) \backslash B^{n}(R)\right)\right) .
$$

However, since $G(R) \subset G \cap\left(B^{n}(R) \times[-R, R]\right)$ and using that the volume element of $G$ is given by $\sqrt{1+|\nabla u|^{2}} d x$ we conclude that

$$
\begin{aligned}
V(G(R)) & \leq \int_{\Omega \cap B^{n}(R) \cap\{|u| \leq R\}} \sqrt{1+|\nabla u|^{2}} \\
& \leq \int_{\Omega \cap B^{n}(R) \cap\{|u| \leq R\}} \frac{|\nabla u|^{2}}{\sqrt{1+|\nabla u|^{2}}}+\int_{\Omega \cap B^{n}(R) \cap\{|u| \leq R\}} \frac{1}{\sqrt{1+|\nabla u|^{2}}} \\
& \leq \frac{1}{\delta} V\left(\Omega \cap\left(B^{n}((1+\delta) R) \backslash B^{n}(R)\right)\right)+V\left(\Omega \cap B^{n}(R)\right) .
\end{aligned}
$$

Using the facts that

$$
V\left(\Omega \cap B^{n}(R)\right) \leq \omega_{n} R^{n}
$$

and

$$
V\left(\Omega \cap\left(B^{n}((1+\delta) R) \backslash B^{n}(R)\right)\right) \leq \omega_{n}\left((1+\delta)^{n} R^{n}-R^{n}\right)
$$

the second assertion follows by letting $\delta \rightarrow 0$.

The following lemma is an adaptation of a well known argument for minimal submanifolds with boundary. For completeness sake, we will give an outline of the proof.

Lemma 2. Let $M^{n}$ be an $n$ dimensional minimal submanifold with boundary $\partial M$ in Euclidean space $\mathbb{R}^{N}$. Let $f$ be a non-negative subharmonic function on $M$ such that $f=0$ on $\partial M$. Then for any point $x \in M$ and $R>0$, we have

$$
f(x) \leq \frac{1}{\omega_{n} R^{n}} \int_{M \cap B(x, R)} f(y) d y
$$

where $B(x, R)$ is the ball in $\mathbb{R}^{N}$ centered at the point $x$ of radius $R$.

Proof. Let $\eta(t)$ be a nonnegative, nonincreasing smooth function on $\mathbb{R}$ with support in the interval $(-\infty, 1)$. Let 


$$
\psi(r)=\int_{r}^{\infty} t \eta(t / s) d t
$$

where $0<s<R$ and $r(y)=r(x, y)$ is the Euclidean distance function. Using the assumption that $M^{n}$ is minimal, we have

$$
\Delta_{M} r^{2}=2 n
$$

and

$$
|\nabla r| \leq 1
$$

on $M$, hence

$$
\Delta_{M} \psi(r) \leq s^{n+1} \frac{\partial}{\partial s}\left(s^{-n} \eta(r / s)\right) .
$$

On the other hand,

$$
\begin{aligned}
\int_{M} f \Delta_{M} \psi & =\int_{M} \psi \Delta_{M} f+\int_{\partial M} f \frac{\partial \psi}{\partial \nu}-\int_{\partial M} \psi \frac{\partial f}{\partial \nu} \\
& \geq 0
\end{aligned}
$$

where $\nu$ is the outward unit normal vector of $\partial M$. In particular, we obtain

$$
\int_{M} f s^{n+1} \frac{\partial}{\partial s}\left(s^{-n} \eta(r / s)\right) \geq 0,
$$

hence

$$
\frac{\partial}{\partial s}\left(s^{-n} \int_{M} f \eta(r / s)\right) \geq 0
$$

and

$$
\frac{1}{\omega_{n} R^{n}} \int_{M} f \eta(r / R) \geq \lim _{s \rightarrow 0} \frac{1}{\omega_{n} s^{n}} \int_{M} f \eta(r / s) .
$$

Letting $\eta(t)$ converge to the characteristic function of the interval $(-\infty, 1)$, the right hand side converges to $f(x)$ and the lemma follows.

We are no ready to prove the main theorem.

Theorem. Suppose $\left\{G_{i}\right\}_{i=1}^{k}$ is a set of $k$ minimal graphs in $\mathbb{R}^{n+1}$. Let us assume that they are defined by the functions $\left\{u_{i}\right\}_{i=1}^{k}$ with disjoint supports $\left\{\Omega_{i}\right\}_{i=1}^{k}$ in $\mathbb{R}^{n}$. Then $k$ must be at most $(n+1) 2^{n}$.

Proof. According to our hypothesis,

$$
G_{i}=\left\{\left(x, u_{i}(x)\right) \in \mathbb{R}^{n+1} \mid x \in \Omega_{i}\right\} .
$$


Let $B^{n+1}(p, R)$ be the Euclidean ball of radius $R$ centered at point $p \in \mathbb{R}^{n+1}$. We will denote

$$
G_{i}(p, R)=G_{i} \cap B^{n+1}(p, R)
$$

to be the extrinsic ball of $G_{i}$ centered at $p$ with radius $R>0$. If $p$ is the origin of $\mathbb{R}^{n+1}$, then we simply write $G_{i}(p, R)$ as $G_{i}(R)$. Let us choose $R_{0}>0$ such that $G_{i}\left(R_{0}\right) \neq \emptyset$ for $i=1, \ldots, k$. Since $\left|u_{i}\right| \leq R$ on $G_{i}(R)$ and $V\left(G_{i}(R)\right) \leq$ $(n+1) \omega_{n} R^{n}$, for any $m \geq 1$, we have

$$
\begin{aligned}
\prod_{j=0}^{m} \prod_{i=1}^{k} \frac{\int_{G_{i}\left(2^{j} R_{0}\right)}\left|u_{i}\right|}{\int_{G_{i}\left(2^{j+1} R_{0}\right)}\left|u_{i}\right|} & =\prod_{i=1}^{k} \frac{\int_{G_{i}\left(R_{0}\right)}\left|u_{i}\right|}{\int_{G_{i}\left(2^{m+1} R_{0}\right)}\left|u_{i}\right|} \\
& \geq \alpha\left((n+1) \omega_{n} 2^{(n+1)(m+1)} R_{0}^{n+1}\right)^{-k}
\end{aligned}
$$

where $\alpha=\prod_{i=1}^{k} \int_{G_{i}\left(R_{0}\right)}\left|u_{i}\right|$. Thus, there exists $0 \leq t \leq m$ such that

$$
\prod_{i=1}^{k} \frac{\int_{G_{i}\left(2^{t} R_{0}\right)}\left|u_{i}\right|}{\int_{G_{i}\left(2^{t+1} R_{0}\right)}\left|u_{i}\right|} \geq \alpha^{\frac{1}{m}}\left((n+1) \omega_{n} 2^{(n+1)(m+1)} R_{0}^{n+1}\right)^{-\frac{k}{m}} .
$$

The arithmetic-geometric means implies

$$
\begin{aligned}
\sum_{i=1}^{k} \frac{\int_{G_{i}\left(2^{t} R_{0}\right)}\left|u_{i}\right|}{\int_{G_{i}\left(2^{t+1} R_{0}\right)}\left|u_{i}\right|} & \geq k\left(\prod_{i=1}^{k} \frac{\int_{G_{i}\left(2^{t} R_{0}\right)}\left|u_{i}\right|}{\int_{G_{i}\left(2^{t+1} R_{0}\right)}\left|u_{i}\right|}\right)^{1 / k} \\
& \geq k \alpha^{\frac{1}{m k}}\left((n+1) \omega_{n} 2^{(n+1)(m+1)} R_{0}^{n+1}\right)^{-\frac{1}{m}}
\end{aligned}
$$

On the other hand, using the fact that the $u_{i}$ 's are disjointly supported, we have

$$
\begin{aligned}
\sum_{i=1}^{k} \frac{\int_{G_{i}\left(2^{t} R_{0}\right)}\left|u_{i}\right|}{\int_{G_{i}\left(2^{t+1} R_{0}\right)}\left|u_{i}\right|} & =\int_{\cup_{i=1}^{k} G_{i}\left(2^{t} R_{0}\right)} \sum_{i=1}^{k} \frac{\left|u_{i}\right|}{\int_{G_{i}\left(2^{t+1} R_{0}\right)}\left|u_{i}\right|} \\
& \leq \max _{x \in \cup_{i=1}^{k} G_{i}\left(2^{t} R_{0}\right)} \sum_{i=1}^{k} \frac{\left|u_{i}(x)\right|}{\int_{G_{i}\left(2^{t+1} R_{0}\right)}\left|u_{i}\right|} \sum_{i=1}^{k} V\left(G_{i}\left(2^{t} R_{0}\right)\right) \\
& =\frac{\left|u_{i_{0}}\left(x_{0}\right)\right|}{\int_{G_{i_{0}}\left(2^{t+1} R_{0}\right)}\left|u_{i_{0}}\right|} \sum_{i=1}^{k} V\left(G_{i}\left(2^{t} R_{0}\right)\right)
\end{aligned}
$$

for some $1 \leq i_{0} \leq k$ and $x_{0} \in G_{i_{0}}\left(2^{t} R_{0}\right)$. However, the assumption that the $u_{i}^{\prime} s$ are disjointly supported and Lemma 1 implies that 


$$
\begin{aligned}
\sum_{i=1}^{k} V & \left(G_{i}\left(2^{t} R_{0}\right)\right) \\
& \leq \sum_{i=1}^{k}\left(V\left(\Omega_{i} \cap B^{n}\left(2^{t} R_{0}\right)\right)+\frac{1}{\delta} V\left(\Omega_{i} \cap\left(B^{n}\left((1+\delta) 2^{t} R_{0}\right) \backslash B^{n}\left(2^{t} R_{0}\right)\right)\right)\right) \\
& \leq \omega_{n}\left(2^{t} R_{0}\right)^{n}+\frac{\omega_{n}}{\delta}\left(\left((1+\delta) 2^{t} R_{0}\right)^{n}-\left(2^{t} R_{0}\right)^{n}\right) \\
& \leq \omega_{n}\left(2^{t} R_{0}\right)^{n}\left(1+\frac{(1+\delta)^{n}-1}{\delta}\right) .
\end{aligned}
$$

Letting $\delta \rightarrow 0$, we conclude that

$$
\sum_{i=1}^{k} V\left(G_{i}\left(2^{t} R_{0}\right)\right) \leq(n+1) \omega_{n}\left(2^{t} R_{0}\right)^{n} .
$$

On the other hand, Lemma 2 implies that

$$
\begin{aligned}
\left|u_{i_{0}}\left(x_{0}\right)\right| & \leq \frac{1}{\omega_{n}\left(2^{t} R_{0}\right)^{n}} \int_{G_{i_{0}} \cap B\left(x_{0}, 2^{t} R_{0}\right)}\left|u_{i_{0}}(y)\right| d y \\
& \leq \frac{1}{\omega_{n}\left(2^{t} R_{0}\right)^{n}} \int_{G_{i_{0}}\left(2^{t+1} R_{0}\right)}\left|u_{i_{0}}(y)\right| d y .
\end{aligned}
$$

Substituting (3) and (4) into (2), we obtain

$$
\sum_{i=1}^{k} \frac{\int_{G_{i}\left(2^{t} R_{0}\right)}\left|u_{i}\right|}{\int_{G_{i}\left(2^{t+1} R_{0}\right)}\left|u_{i}\right|} \leq n+1 .
$$

Combining with the inequality (1) we obtain

$$
k \alpha^{\frac{1}{m k}}\left((n+1) \omega_{n} 2^{(n+1)(m+1)} R_{0}^{n+1}\right)^{-\frac{1}{m}} \leq n+1 .
$$

Letting $m \rightarrow \infty$, this gives

$$
k \leq(n+1) 2^{n+1}
$$

as claimed.

Corollary. Let $G \subset \mathbb{R}^{n+1}$ be an entire minimal graph defined on $\mathbb{R}^{n}$ given by the function $u$. Then $G \backslash \mathbb{R}^{n}$ must have at most $(n+1) 2^{n+1}$ components.

We would also like to point out that the Theorem implies that if $D$ is a noncompact domain such that more than $(n+1) 2^{n+1}$ disjoint copies can be fit inside $\mathbb{R}^{n}$, then it will not support a minimal graph. 


\section{References}

[E-R] R. Sa Earp and H. Rosenberg, The Dirichlet problem for the minimal surface equation on unbounded planar domains, J. Math. Pures Appl. (9) 68 (1989), no. 2, 163-183.

[L] P. Li, Harmonic sections of polynomial growth, Math. Res. Lett. 4 (1997), no. 1, 35-44.

[L-W] P. Li and J. Wang, Convex hull properties of harmonic maps, J. Differential Geom. 48 (1998), no. 3, 497-530.

[M-R] W. Meeks III and H. Rosenberg, The uniqueness of the helicoid and the asymptotic geometry of perperly embedded minimal surfaces with finite topology, preprint.

Department of Mathematics, University of California, Irvine, CA 92697-3875.

E-mail address: pli@math.uci.edu

School of Mathematics, University of Minnesota, Minneapolis, MN 55455.

E-mail address: jiaping@math.umn.edu 